\title{
Modular adjacency algebras, standard representations, and $p$-ranks of cyclotomic association schemes
}

\author{
Akihide Hanaki *†
}

\begin{abstract}
In this paper, we consider cyclotomic association schemes $S=\operatorname{Cyc}\left(p^{a}, d\right)$. We focus on the adjacency algebra of $S$ over algebraically closed fields $K$ of characteristic $p$. If $p \equiv 1(\bmod d), p \equiv-1(\bmod d)$, or $d \in\{2,3,4,5,6\}$, we identify the adjacency algebra of $S$ over $K$ as a quotient of a polynomial ring over an admissible ideal. In several cases, we determine the indecomposable direct sum decomposition of the standard module of $S$. As a consequence, we are able to compute the $p$-rank of several specific elements of the adjacency algebra of $S$ over $K$.
\end{abstract}

\section{Introduction}

Two association schemes are said to be algebraically isomorphic if the intersection numbers coincide. In this case, their algebraic properties are the same but combinatorial properties are not, in general. For example, distance-regular graphs with the same intersection array give algebraically isomorphic association schemes. In general, it is not so easy to distinguish them. In some papers, for example $[3,7,9,10]$, it was shown that $p$-ranks, ranks of matrices over a field of characteristic $p$, of adjacency matrices can distinguish algebraically isomorphic association schemes for some examples. In [7], Yoshikawa and the author considered the structure of adjacency algebras and standard modules (representations) over a field of characteristic $p$ and relation with the $p$-ranks. Structures of modular adjacency algebras were studied in $[11,12,13]$.

In this paper, we consider the adjacency algebra and representations of the cyclotomic (association) scheme $\operatorname{Cyc}\left(p^{a}, d\right)$ over a field of characteristic $p$. We will give an effective method to determine the structure of the adjacency algebra (Theorem 3.4). In general, the structure seems to be complicated. So we will give concrete structures for some special cases $: p \equiv 1(\bmod d)($ Theorem 3.7$), p \equiv-1(\bmod d)$ (Theorem 3.8),

\footnotetext{
*Faculty of Science, Shinshu University, Matsumoto, 390-8621, Japan, e-mail : hanaki@shinshuu.ac.jp

${ }^{\dagger}$ This work was supported by JSPS KAKENHI Grant Number 25400011.
} 
and $d=2,3,4,5,6$ (theorems in Subsection 3.4). We will describe the algebra as a quotient of a polynomial ring by an admissible ideal. Also we determine the structures of standard modules for the case $p \equiv 1(\bmod d)$ (Theorem 4.2$)$ and $d=3$ (Theorem 4.6). We will determine the indecomposable direct sum decomposition of the module. For these cases, we also determine the $p$-ranks of some matrices (Corollaries 4.4 and 4.7) and compare them with some algebraically isomorphic association schemes (Examples $4.5,4.8$, and 4.9).

A cyclotomic scheme is defined by the action of a subgroup $G$ of the affine group $\operatorname{AGL}\left(1, p^{a}\right)$. The standard module is a $G$-module and its $G$-submodules are linear codes invariant under $G$. Linear codes invariant under AGL $\left(1, p^{a}\right)$ were classified in [8].

\section{Preliminaries}

\subsection{Association schemes and adjacency algebras}

Following the book [1] or [14], we will define association schemes and adjacency algebras.

Let $X$ be a finite set. For $s \subset X \times X$, we define a matrix $\sigma_{s}$ whose rows and columns are indexed by the set $X$ and the $(x, y)$-entry of $\sigma_{s}$ is 1 if $(x, y) \in s$ and 0 otherwise. We call $\sigma_{s}$ the adjacency matrix of $s$. Let $X \times X=\bigcup_{s \in S} s$ be a partition. We call the pair $(X, S)$ an association scheme if the following three conditions hold :

(1) The diagonal relation $\{(x, x) \mid x \in X\}$ is in $S$, we denote it by 1 .

(2) For every $s \in S$, the transposition $\{(y, x) \mid(x, y) \in s\}$ is in $S$, we denote it by $s^{*}$.

(3) For all $s, t, u \in S$, there are non-negative integers $p_{s t}^{u}$ such that $\sigma_{s} \sigma_{t}=\sum_{u \in S} p_{s t}^{u} \sigma_{u}$, where the product is the usual matrix product.

The number $p_{s t}^{u}$ in the condition (3) is called an intersection number. For $s \in S$, we call $n_{s}=p_{s s^{*}}^{1}$ the valency of $s$. Two association schemes $(X, S)$ and $\left(X^{\prime}, S^{\prime}\right)$ are isomorphic if there are bijections $\varphi: X \rightarrow X^{\prime}$ and $\psi: S \rightarrow S^{\prime}$ such that $(x, y) \in s$ if and only if $(\varphi(x), \varphi(y)) \in \psi(s)$. They are algebraically isomorphic if there is a bijection $\psi: S \rightarrow S^{\prime}$ such that $p_{s t}^{u}=p_{\psi(s) \psi(t)}^{\psi(u)}$ for all $s, t, u \in S$.

Let $K$ be a field. We regard $\sigma_{s}(s \in S)$ as matrices over $K$. Then, by the condition (3), we can define a $K$-algebra $K S=\bigoplus_{s \in S} K \sigma_{s}$ of dimension $|S|$. We call the algebra $K S$ the adjacency algebra of $(X, S)$ over $K$. We regard $K S$ as a subalgebra of the full matrix algebra $\operatorname{Mat}_{X}(K)$.

Let $K X$ be the $K$-vector space with basis $X$. Since we regard $K S$ as a subalgebra of $\operatorname{Mat}_{X}(K)$, we can see that $K X$ is a right $K S$-module. We call this module the standard $K S$-module. The corresponding representation is $K S \rightarrow \operatorname{Mat}_{X}(K)\left(\sigma_{s} \mapsto \sigma_{s}\right)$, and we call this the standard representation of $(X, S)$ over $K$. 


\subsection{Schurian (association) schemes}

Let $G$ be a finite transitive permutation group on a finite set $X$. Then $G$ also acts on $X \times X$ diagonally. The set $S$ of orbits of $G$ on $X \times X$ define a partition of $X \times X$. It is known that $(X, S)$ becomes an association scheme [1, II, Example 2.1]. An association scheme obtained in this way is said to be schurian. For $x \in X$, let $H=G_{x}$ be the stabilizer of $x$ in $G$. Then we can identify $X$ with $H \backslash G$. So we can say that a schurian scheme is obtained by a finite group and its subgroup. We denote it by $\mathfrak{X}(G, H)$.

For a schurian scheme, the adjacency algebra is understood by the following way. Let $T$ be the permutation representation of $G$ over a field $K$. Then the adjacency algebra is just the centralizer algebra $\left\{A \in \operatorname{Mat}_{X}(K) \mid A T(g)=T(g) A\right.$ for any $\left.g \in G\right\}$.

Let $N$ be a finite group, and let $H$ be a subgroup of the automorphism group of $N$. We can define the semidirect product $N \rtimes H$. We consider the schurian scheme $(X, S)=\mathfrak{X}(N \rtimes H, H)$. For this case, $N$ is a regular normal subgroup of $N \rtimes H$ and we can apply [1, II, Theorem 6.1]. Let $K$ be a field. The group $H$ also acts on the group algebra $K N$. By [1, II, Theorem 6.1], the adjacency algebra is just the ring of fixed points $(K N)^{H}=\left\{\alpha \in K N \mid \alpha^{h}=\alpha\right.$ for any $\left.h \in H\right\}$, where we regard $K N$ as a subalgebra of $\operatorname{Mat}_{N}(K)$ by a regular permutation representation. Since $(K N)^{H} \subset K N$, $K N$ becomes a right $(K N)^{H}$-module. This is just the standard module of the scheme $\mathfrak{X}(N \rtimes H, H)$.

\subsection{Cyclotomic (association) schemes}

Let $p$ be a prime number. We denote the finite field of $p^{a}$ elements by $\mathbb{F}_{p^{a}}$. The multiplicative group $\mathbb{F}_{p^{a}}^{\times}=\mathbb{F}_{p^{a}} \backslash\{0\}$ is a cyclic group of order $p^{a}-1$. We fix a primitive element $\zeta$ of $\mathbb{F}_{p^{a}}$ (a generator of $\mathbb{F}_{p^{a}}^{\times}$). Let $N$ be an elementary abelian group of order $p^{a}$ and fix a group isomorphism $\mathbb{F}_{p^{a}} \rightarrow N(\alpha \mapsto[\alpha])$. Let $H=\left\langle h_{0}\right\rangle$ be a cyclic group of order $p^{a}-1$ and define the action of $H$ on $N$ by $[\alpha]^{h_{0}}=[\zeta \alpha]$. Let $d$ be a divisor of $p^{a}-1$. There is a unique subgroup $H_{d}=\left\langle h_{0}^{d}\right\rangle$ of $H$ of index $d$. Now we can define a schurian scheme $\mathfrak{X}\left(N \rtimes H_{d}, H_{d}\right)$. We call this the cyclotomic (association) scheme and denote it by $\operatorname{Cyc}\left(p^{a}, d\right)$. For details, see [4].

Adjacency matrices of $\operatorname{Cyc}\left(p^{a}, d\right)$ are obtained as follows. Put $X_{0}=\{0\}$ and $X_{i}=$ $\left\{\zeta^{j} \mid 0 \leq j<p^{a}, j \equiv i(\bmod d)\right\}(i=1, \ldots, d)$. Then $X_{0}, X_{1}, \ldots, X_{d}$ are $H_{d^{-}}$orbits of $\mathbb{F}_{p^{a}}$. Let $T$ be a regular permutation representation of $N$. Then the adjacency matrices are

$$
\sigma_{i}=\sum_{\alpha \in X_{i}} T(\alpha) \quad(i=0,1, \ldots, d) .
$$

We prove one easy lemma here.

Lemma 2.1. Let $s$ and $t$ be non-trivial relations of the cyclotomic scheme $\operatorname{Cyc}\left(p^{a}, d\right)$, and let $f$ be a polynomial over an arbitrary field $K$. The $f\left(\sigma_{s}\right)$ and $f\left(\sigma_{t}\right)$ have the same rank over $K$. 
Proof. Let $P$ be the permutation matrix on $N$ defined by the multiplication of $\zeta$. Then $P^{-1} \sigma_{i} P=\sigma_{i+1}$ for $i=1, \ldots, d-1$ and $P^{-1} \sigma_{d} P=\sigma_{1}$, where $\sigma_{i}$ are defined as above. So the lemma holds.

\section{$3 \quad$ Adjacency algebras of cyclotomic schemes}

Let $K$ be an algebraically closed field of characteristic $p$. In this section, we will give an effective method to determine the structure of the adjacency algebra of a cyclotomic scheme $\operatorname{Cyc}\left(p^{a}, d\right)$ over $K$. In general, the structure seems to be complicated. So we will give concrete structures for some spacial cases in Subsections 3.2, 3.3, and 3.4.

We use notations in Subsection 2.3. First, we will consider the $K H$-module structure of $K N$. The following facts are well known.

(1) $K N$ is a local algebra with the Jacobson radical $\left\{\sum_{n \in N} c_{n} n \mid \sum_{n \in N} c_{n}=0\right\}$.

(2) Let $\left\{n_{1}, \ldots, n_{a}\right\}$ be a set of generators of $N$, and put $x_{i}=n_{i}-1$ for $i=1, \ldots, a$. Then $K N=K\left[x_{1}, \ldots, x_{a}\right]$ with relations $x_{i}^{p}=0(i=1, \ldots, a)$.

We remark that the group $H=\left\langle h_{0}\right\rangle \cong C_{p^{a}-1}$ is a $p^{\prime}$-group and so $K H$ is semisimple. Since $J(K N)$ is fixed by automorphisms, we have

$$
K N \cong \bigoplus_{i=1}^{a(p-1)} J^{i-1}(K N) / J^{i}(K N)
$$

as a $K H$-module. We consider $J(K N) / J^{2}(K N)$. It has basis $\left\{\overline{x_{i}} \mid i=1, \ldots, a\right\}$, where $\overline{x_{i}}=x_{i}+J^{2}(K N)$. Put $\alpha_{i} \in \mathbb{F}_{p^{a}}$ with $\left[\alpha_{i}\right]=n_{i}$ for $i=1, \ldots, a$.

Lemma 3.1. We have $\left[\sum_{i=1}^{a} c_{i} \alpha_{i}\right]-[0] \equiv \sum_{i=1}^{a} c_{i} x_{i}\left(\bmod J^{2}(K N)\right)$ for $c_{i} \in \mathbb{F}_{p}(i=$ $1, \ldots, a)$.

Proof. Remark that $[\alpha][\beta]=[\alpha+\beta]$. So $[c \alpha]=[\alpha]^{c}$ for a non-negative integer $c$.

We show that $\left[c \alpha_{i}\right]-[0] \equiv c x_{i}\left(\bmod J^{2}(K N)\right)$ by induction on $0 \leq c<p$. If $c=0,1$, then this is clear. Suppose $2 \leq c<p$. By inductive hypothesis, we have

$$
\begin{aligned}
{\left[c \alpha_{i}\right]-[0] } & =\left[\alpha_{i}\right]^{c}-[0]=\left(\left[\alpha_{i}\right]-[0]\right)\left(\left[\alpha_{i}\right]^{c-1}+\cdots+\left[\alpha_{i}\right]+[0]\right) \\
& =x_{i}\left(\left(\left[\alpha_{i}\right]^{c-1}-[0]\right)+\cdots+\left(\left[\alpha_{i}\right]-[0]\right)+c[0]\right) \equiv c x_{i} \quad\left(\bmod J^{2}(K N)\right) .
\end{aligned}
$$

Now we have

$$
\begin{aligned}
{\left[\sum_{i=1}^{a} c_{i} \alpha_{i}\right] } & =\prod_{i=1}^{a}\left[c_{i} \alpha_{i}\right] \equiv \prod_{i=1}^{a}\left(c_{i} x_{i}+[0]\right) \\
& \equiv \sum_{i=1}^{a} c_{i} x_{i}+[0] \quad\left(\bmod J^{2}(K N)\right)
\end{aligned}
$$

The assertion holds. 
Lemma 3.2. Recall that $\zeta$ is a primitive element of $\mathbb{F}_{p^{a}}$ and $h_{0}$ is a generator of $H$. Put $\alpha_{i} \zeta=\sum_{j=1}^{n} c_{i j} \alpha_{j}\left(c_{i j} \in \mathbb{F}_{p}\right)$. Namely $\zeta \mapsto\left(c_{i j}\right)$ is a regular representation of $\mathbb{F}_{p^{a}}$ over $\mathbb{F}_{p}$. Then $x_{i}^{h_{0}} \equiv \sum_{j=1}^{n} c_{i j} x_{j}\left(\bmod J^{2}(K N)\right)$. This means that $J(K N) / J^{2}(K N)$ is a KH-module given by the regular representation of $H \cong \mathbb{F}_{p^{a}}^{\times}$.

Proof. By Lemma 3.1, we have $x_{i}^{h_{0}}=\left[\alpha_{i} \zeta\right]-[0]=\left[\sum_{j=1}^{a} c_{i j} \alpha_{j}\right]-[0] \equiv \sum_{j=1}^{a} c_{i j} x_{j}$.

Lemma 3.3. There exist $v_{1}, \ldots, v_{a} \in J(K N)$ such that the following statements holds.

(1) The set $\left\{v_{1}^{f_{1}} \cdots v_{a}^{f_{a}}+J^{\ell}(K N) \mid 0 \leq f_{i}<p(1 \leq i \leq a), \sum_{i=1}^{a} f_{i}=\ell\right\}$ is a basis of $J^{\ell}(K N) / J^{\ell+1}(K N)$ for $\ell=0,1, \ldots, a(p-1)$. Hence $\left\{v_{1}^{f_{1}} \cdots v_{a}^{f_{a}} \mid 0 \leq f_{i}<p(1 \leq\right.$ $i \leq a)\}$ is a basis of $K N$.

(2) If $0 \leq f_{i}<p$ for all $1 \leq i \leq a$, then $K v_{1}^{f_{1}} \cdots v_{a}^{f_{a}}$ is a one-dimensional right $K H$ module such that $\left(v_{1}^{f_{1}} \cdots v_{a}^{f_{a}}\right)^{h_{0}}=\zeta^{f} v_{1}^{f_{1}} \cdots v_{a}^{f_{a}}$, where $f=\sum_{i=1}^{a} f_{i} p^{i-1}$.

Proof. As in Lemma 3.2, $J(K N) / J^{2}(K N)$ is an $K N$-module given by the regular representation of $\mathbb{F}_{p^{a}}^{\times}$over $\mathbb{F}_{p}$. Define $C=\left(c_{i j}\right)$ as in the proof of Lemma 3.2. Since $\zeta$ is an eigenvalue of $C$, their conjugates $\zeta^{p}, \zeta^{p^{2}}, \ldots, \zeta^{p^{a-1}}$ are also eigenvalues of $C$. Since $K H$ is semisimple and commutative, we have $K N \cong K N / J(K N) \oplus J(K N) / J^{2}(K N) \oplus J^{2}(K N)$ as a $K H$-module and $J(K N) / J^{2}(K N)$ is a direct sum of one dimensional submodules. There are $v_{1}, \ldots, v_{a} \in J(K N)$ such that $\left\{v_{1}, \ldots, v_{a}\right\}$ is a $K$-basis of $J(K N) / J^{2}(K N)$ and $v_{i}^{h_{0}}=\zeta^{p^{i-1}} v_{i}$. This shows that (1) holds for $\ell=1$.

Since $v_{i}^{p}=0(i=1, \ldots, a)$ and $v_{1}, \ldots, v_{a}$ generate $K N$, we can see that $v_{i}^{p-1} \neq 0$ $(i=1, \ldots, a)$. It is easy to see that the all assertions hold.

We use the elements $v_{1}, \ldots, v_{a}$ obtained in Lemma 3.3 throughout this paper. We fix one more notation. For $0 \leq f \leq p^{a}-1$, we can write

$$
f=\sum_{i=1}^{a} f_{i} p^{i-1}, \quad 0 \leq f_{i} \leq p-1 \quad(i=1, \ldots, a)
$$

uniquely. We put

$$
\boldsymbol{v}^{(f)}=v_{1}^{f_{1}} \cdots v_{a}^{f_{a}} .
$$

Then Lemma 3.3 says that $\left\{\boldsymbol{v}^{(f)} \mid 0 \leq f \leq p^{a}-1\right\}$ is a basis of $K N$.

Now we can show the most important theorem in this section.

Theorem 3.4. We consider a cyclotomic scheme $(X, S)=\operatorname{Cyc}\left(p^{a}, d\right)=\mathfrak{X}\left(N \rtimes H_{d}, H_{d}\right)$, where $d \mid p^{a}-1$. Let $K$ be an algebraically closed field of characteristic $p$. Put $e=$ $\left(p^{a}-1\right) / d$. Then $\left\{\boldsymbol{v}^{(i e)} \mid 0 \leq i \leq d\right\}$ is a basis of the adjacency algebra KS. The product $\boldsymbol{v}^{(i e)} \boldsymbol{v}^{(j e)}$ is $\boldsymbol{v}^{((i+j) e)}$ or 0 .

Proof. By definition, we know that $\operatorname{dim}_{K} K S=|S|=d+1$. So it is enough to show that $\boldsymbol{v}^{(i e)}$ is fixed by $H_{d}=\left\langle h_{0}^{d}\right\rangle$. By Lemma $3.3(2)$, we have

$$
\left(\boldsymbol{v}^{(i e)}\right)^{h_{0}^{d}}=\zeta^{i e d} \boldsymbol{v}^{(i e)}=\zeta^{i\left(p^{a}-1\right)} \boldsymbol{v}^{(i e)}=\boldsymbol{v}^{(i e)} .
$$

The last statement is clear. 
We determine the expression $i e=\sum_{j=1}^{a} f_{j} p^{j-1}$ for $i=0,1, \ldots, d$. For an integer $m$, we denote by $\rho(m)$ the smallest non-negative integer such that $m \equiv \rho(m)(\bmod d)$. Put

$$
\alpha_{m}=\frac{\rho(m) p-\rho(m p)}{d} .
$$

Obviously $\alpha_{m}=\alpha_{m^{\prime}}$ if $m \equiv m^{\prime}(\bmod d)$.

Lemma 3.5. For every integer $m, \alpha_{m}$ is a non-negative integer and $0 \leq \alpha_{m}<p$. We have

$$
i e=\sum_{j=1}^{a} \alpha_{i p^{a-j}} p^{j-1}
$$

for $i=1, \ldots, d-1$, and for $i=0, d$,

$$
0 e=\sum_{j=1}^{a} 0 p^{j-1}, \quad d e=\sum_{j=1}^{a}(p-1) p^{j-1} .
$$

Proof. By the definition of $\rho, \alpha_{m}$ is a non-negative integer and $0 \leq \alpha_{m}<p$. The equations for $i=0, d$ are trivial. Suppose that $0<i<d$. Remark that $\rho(i)=i$ and $p^{a} \equiv 1(\bmod d)$. We have

$$
\begin{aligned}
\sum_{j=1}^{a} \alpha_{i p^{a-j}} p^{j-1} & =\sum_{j=1}^{a} \frac{\rho\left(i p^{a-j}\right) p-\rho\left(i p^{a-j+1}\right)}{d} p^{j-1} \\
& =\frac{\rho(i) p}{d} p^{a-1}+\sum_{j=1}^{a-1} \frac{\rho\left(i p^{a-j}\right) p}{d} p^{j-1}-\sum_{j=2}^{a} \frac{\rho\left(i p^{a-j+1}\right)}{d} p^{j-1}-\frac{\rho\left(i p^{a}\right)}{d} \\
& =\frac{\rho(i) p^{a}}{d}-\frac{\rho\left(i p^{a}\right)}{d}=\frac{i\left(p^{a}-1\right)}{d}=i e .
\end{aligned}
$$

Now the lemma holds.

We remark that $\boldsymbol{v}^{(i e)} \boldsymbol{v}^{(j e)}=\boldsymbol{v}^{((i+j) e)} \neq 0$ if and only if $\alpha_{i p^{a-\ell}}+\alpha_{j p^{a-\ell}}<p$ for all $\ell=1, \ldots, a$ by Lemma 3.5. Easily we can see that $\boldsymbol{v}^{(0)} \boldsymbol{v}^{(i e)}=\boldsymbol{v}^{(i e)} \neq 0, \boldsymbol{v}^{(i e)} \boldsymbol{v}^{((d-i) e)}=$ $\boldsymbol{v}^{(d e)} \neq 0$, and $\boldsymbol{v}^{(i e)} \boldsymbol{v}^{(j e)}=0$ if $i+j>d$. By Theorem 3.4, it is easy to determine the structure of the adjacency algebra for a given parameters $p^{a}$ and $d$. But it seems to be complicated to state the structure in general. So we determine the structure for some special cases.

We denote by $K \operatorname{Cyc}\left(p^{a}, d\right)$ the adjacency algebra of $\operatorname{Cyc}\left(p^{a}, d\right)$ over a field $K$. We remark that $K \mathrm{Cyc}\left(p^{a}, d\right)$ is a commutative local symmetric algebra if the characteristic of $K$ is $p$ by [5].

\section{1 $K \mathrm{Cyc}\left(p^{a}, d\right)$ and $K \mathrm{Cyc}\left(p^{b}, d\right)$ are isomorphic}

For coprime numbers $d$ and $p$, we denote by $\operatorname{ord}_{d}(p)$ the smallest positive integer such that $p^{\operatorname{ord}_{d}(p)} \equiv 1(\bmod d)$. Then $p^{a} \equiv 1(\bmod d)$ if and only if $\operatorname{ord}_{d}(p) \mid a$.

We will show the next theorem. 
Theorem 3.6. Let $K$ be an algebraically closed field of characteristic $p$. Suppose that $d \mid p^{a}-1$ and $d \mid p^{b}-1$. Then $K \operatorname{Cyc}\left(p^{a}, d\right) \cong K \operatorname{Cyc}\left(p^{b}, d\right)$.

Proof. We may suppose that $a=\operatorname{ord}_{d}(p)$ and $a \mid b$. Put $e=\left(p^{a}-1\right) / d$ and $e^{\prime}=$ $\left(p^{b}-1\right) / d$. For $j=0,1, \ldots, d$, write

$$
j e=\sum_{i=0}^{a-1} e_{i, j} p^{i}, \quad j e^{\prime}=\sum_{i=0}^{b-1} e_{i, j}^{\prime} p^{i}
$$

where $0 \leq e_{i, j}, e_{i, j}^{\prime} \leq p-1$. Then

$$
\sum_{\ell=0}^{b / a-1} \sum_{i=0}^{a-1} e_{i, j} p^{\ell a+i}=\sum_{\ell=0}^{b / a-1} p^{\ell a} \sum_{i=0}^{a-1} e_{i, j} p^{i}=\frac{p^{b}-1}{p^{a}-1} \cdot j e=j \cdot \frac{p^{b}-1}{p^{a}-1} \cdot \frac{p^{a}-1}{d}=j e^{\prime} .
$$

So

$$
e_{\ell n+i, j}^{\prime}=e_{i, j}
$$

for $0 \leq i \leq a-1$ and $0 \leq \ell \leq b / a-1$ by uniqueness of the expression. Now it is easy to see that $\boldsymbol{v}^{(j e)} \mapsto \boldsymbol{v}^{\left(j e^{\prime}\right)}$ is an isomorphism, where $\left\{\boldsymbol{v}^{(j e)}\right\}$ and $\left\{\boldsymbol{v}^{\left(j e^{\prime}\right)}\right\}$ are bases of $K \mathrm{Cyc}\left(p^{a}, d\right)$ and $K \operatorname{Cyc}\left(p^{b}, d\right)$, respectively.

By Theorem 3.6, it is enough to consider $K \mathrm{Cyc}\left(p^{\operatorname{ord}_{d}(p)}, d\right)$ to determine the structure of the adjacency algebra $K \mathrm{Cyc}\left(p^{a}, d\right)$.

Remark. In [12], Yoshikawa proved that adjacency algebras of Hamming schemes are isomorphic for some parameters. For this case, intersection numbers (structure constants) $p_{s t}^{u}$ are congruent modulo $p$.

For Theorem 3.6, intersection numbers are not necessarily congruent modulo $p$. For example, consider intersection numbers of $\mathrm{Cyc}(7,3)$ and $\mathrm{Cyc}\left(7^{2}, 3\right)$ modulo 7 .

\section{2 $K \operatorname{Cyc}\left(p^{a}, d\right)$ with $p \equiv 1(\bmod d)$}

Theorem 3.7. Let $K$ be an algebraically closed field of characteristic $p$. Suppose that $p \equiv 1(\bmod d)$. Then $K \operatorname{Cyc}\left(p^{a}, d\right) \cong K[x] /\left(x^{d+1}\right)$.

Proof. We may assume that $a=\operatorname{ord}_{d}(p)=1$. Put $e=(p-1) / d$. Then $\boldsymbol{v}^{(i e)}=v_{1}^{i e}$ for $i=1,2, \ldots, d$ and we have the result.

\section{3 $K \operatorname{Cyc}\left(p^{a}, d\right)$ with $p \equiv-1(\bmod d)$}

Theorem 3.8. Let $K$ be an algebraically closed field of characteristic $p$. Suppose that $d \neq 2$ and $p \equiv-1(\bmod d)$. Then $K \mathrm{Cyc}\left(p^{a}, d\right) \cong K\left[x_{1}, \ldots, x_{d-1}\right] / I$, where $I$ is the ideal generated by

$$
x_{i} x_{j} \quad(i+j \neq d), \quad x_{i} x_{j}-x_{k} x_{\ell} \quad(i+j=k+\ell=d) .
$$


Proof. We may assume that $a=\operatorname{ord}_{d}(p)=2$. In Lemma 3.5, put

$$
\alpha_{i}=\frac{i p-(d-i)}{d}
$$

for $i=1,2, \ldots, d-1$. Then $\alpha_{i}$ is an integer and $1 \leq \alpha_{i} \leq p-1$ by $p \equiv-1(\bmod d)$. Easily we have $\alpha_{i}+\alpha_{d-i} p=i e$, where $e=\left(p^{2}-1\right) / d$. So $\boldsymbol{v}^{(i e)}=v_{1}^{\alpha_{i}} v_{2}^{\alpha_{d-i}}$. Since $\alpha_{i}+\alpha_{d-i}=p-1, \boldsymbol{v}^{(i e)} \boldsymbol{v}^{(j e)}=0$ if $i+j \neq d$ and $\boldsymbol{v}^{(i e)} \boldsymbol{v}^{((d-i) e)}=\boldsymbol{v}^{(d e)}(\neq 0)$. Comparing the dimensions, we have the result.

Remark. The cases $p \equiv \pm 1(\bmod d)$ give two extreme structures of $K \operatorname{Cyc}\left(p^{a}, d\right)$. By Theorem 3.4, $K \operatorname{Cyc}\left(p^{a}, d\right)$ is determined by $\boldsymbol{v}^{(i e)} \boldsymbol{v}^{(j e)}(0 \leq i, j \leq d)$. Always $\boldsymbol{v}^{(0)} \boldsymbol{v}^{(j e)}=$ $\boldsymbol{v}^{(j e)} \neq 0, \boldsymbol{v}^{(i e)} \boldsymbol{v}^{((d-i) e)}=\boldsymbol{v}^{(d e)} \neq 0$, and $\boldsymbol{v}^{(i e)} \boldsymbol{v}^{(j e)}=0$ if $i+j>d$. We proved

(1) $\boldsymbol{v}^{(i e)} \boldsymbol{v}^{(j e)} \neq 0$ for all $i+j<d$, if $p \equiv 1(\bmod d)$, and

(2) $\boldsymbol{v}^{(i e)} \boldsymbol{v}^{(j e)}=0$ for all $i \geq 1, j \geq 1, i+j<d$, if $p \equiv-1(\bmod d)$.

Easily we can see that $(1)$ occurs only if $p \equiv 1(\bmod d)$. But $(2)$ can occur for the other case (for example, see Theorem 3.12).

\section{4 $K \operatorname{Cyc}\left(p^{a}, d\right)$ for $d=2,3,4,5,6$}

In this subsection, we will determine the structure of $\operatorname{Cyc}\left(p^{a}, d\right)$ for $d=2,3,4,5,6$. They are not so difficult by Theorems 3.4, 3.6, 3.7, and 3.8.

Theorem 3.9 (Case $d=2$ ). Let $K$ be an algebraically closed field of characteristic $p$. For an odd prime $p$, we have $K \operatorname{Cyc}\left(p^{a}, 2\right) \cong K[x] /\left(x^{3}\right)$.

Proof. For this case, $2 \mid p-1$. So we can apply Theorem 3.7.

Theorem 3.10 (Case $d=3$ ). Let $K$ be an algebraically closed field of characteristic $p$.

(1) If $p \equiv 1(\bmod 3)$, then $K \operatorname{Cyc}\left(p^{a}, 3\right) \cong K[x] /\left(x^{4}\right)$.

(2) If $p \equiv 2(\bmod 3)$, then

$$
K \operatorname{Cyc}\left(p^{a}, 3\right) \cong K[x, y] /\left(x^{2}, y^{2}\right) .
$$

Proof. (1) holds by Theorem 3.7. (2) holds by Theorem 3.8.

Theorem 3.11 (Case $d=4)$. Let $K$ be an algebraically closed field of characteristic $p$.

(1) If $p \equiv 1(\bmod 4)$, then $K \operatorname{Cyc}\left(p^{a}, 4\right) \cong K[x] /\left(x^{5}\right)$.

(2) If $p \equiv 3(\bmod 4)$, then

$$
K \mathrm{Cyc}\left(p^{a}, 4\right) \cong K[x, y, z] /\left(x^{2}, y^{3}, z^{2}, x z, y z\right) .
$$


Proof. (1) holds by Theorem 3.7. (2) holds by Theorem 3.8.

Theorem 3.12 (Case $d=5$ ). Let $K$ be an algebraically closed field of characteristic $p$.

(1) If $p \equiv 1(\bmod 5)$, then $K \operatorname{Cyc}\left(p^{a}, 5\right) \cong K[x] /\left(x^{6}\right)$.

(2) If $p \equiv 2,3,4(\bmod 5)$, then

$$
K \operatorname{Cyc}\left(p^{a}, 5\right) \cong K[x, y, z, u] /\left(x^{2}, y^{2}, z^{2}, u^{2}, x y, x z, y u, z u, x u-y z\right) .
$$

Proof. (1) holds by Theorem 3.7. For the case $p \equiv 4(\bmod 5)$, we can apply Theorem 3.8.

Suppose $p \equiv 2(\bmod 5)$. We may assume that $a=\operatorname{ord}_{5}(p)=4$. Put

$$
\alpha_{1}=\frac{p-2}{5}, \quad \alpha_{2}=\frac{2 p-4}{5}, \quad \alpha_{3}=\frac{3 p-1}{5}, \quad \alpha_{4}=\frac{4 p-3}{5} .
$$

Then

$$
\begin{aligned}
e=\alpha_{3}+\alpha_{4} p+\alpha_{2} p^{2}+\alpha_{1} p^{3}, & 2 e=\alpha_{1}+\alpha_{3} p+\alpha_{4} p^{2}+\alpha_{2} p^{3}, \\
3 e=\alpha_{4}+\alpha_{2} p+\alpha_{1} p^{2}+\alpha_{3} p^{3}, & 4 e=\alpha_{2}+\alpha_{1} p+\alpha_{3} p^{2}+\alpha_{4} p^{3} .
\end{aligned}
$$

We can check the relations.

Suppose $p \equiv 3(\bmod 5)$. We may assume that $a=\operatorname{ord}_{5}(p)=4$. Put

$$
\alpha_{1}=\frac{p-3}{5}, \quad \alpha_{2}=\frac{2 p-1}{5}, \quad \alpha_{3}=\frac{3 p-4}{5}, \quad \alpha_{4}=\frac{4 p-2}{5} .
$$

Then

$$
\begin{aligned}
e=\alpha_{2}+\alpha_{4} p+\alpha_{3} p^{2}+\alpha_{1} p^{3}, & 2 e=\alpha_{4}+\alpha_{3} p+\alpha_{1} p^{2}+\alpha_{2} p^{3}, \\
3 e=\alpha_{1}+\alpha_{2} p+\alpha_{4} p^{2}+\alpha_{3} p^{3}, & 4 e=\alpha_{3}+\alpha_{1} p+\alpha_{2} p^{2}+\alpha_{4} p^{3} .
\end{aligned}
$$

We can check the relations.

Theorem 3.13 (Case $d=6$ ). Let $K$ be an algebraically closed field of characteristic $p$.

(1) If $p \equiv 1(\bmod 6)$, then $K \operatorname{Cyc}\left(p^{a}, 6\right) \cong K[x] /\left(x^{7}\right)$.

(2) If $p \equiv 5(\bmod 6)$, then

$$
K \operatorname{Cyc}\left(p^{a}, 6\right) \cong K[x, y, z, u, v] / I
$$

where $I$ is an ideal generated by $x^{2}, y^{2}, z^{3}, u^{2}, v^{2}, x y, x z, x u, y z, y v, z u, z v$, $x v-z^{2}, y u-z^{2}$.

Proof. (1) holds by Theorem 3.7. (2) holds by Theorem 3.8 . 


\section{Standard modules of cyclotomic schemes and $p$ - ranks}

In this section, we will consider structures of standard modules and $p$-ranks of elements of adjacency algebras. Let $(X, S)=\operatorname{Cyc}\left(p^{a}, d\right)=\mathfrak{X}\left(N \rtimes H_{d}, H_{d}\right)$ be a cyclotomic scheme, and let $K$ be an algebraically closed field of characteristic $p$. We recall that the adjacency algebra is $K S=(K N)^{H_{d}}$ and the standard module is $K X=K N$. So we

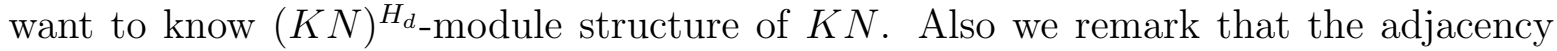
algebra $K S$ is local. So, if an element of $K S$ is not in the Jacobson radical, then it is invertible and has full rank. We are interested in elements in the Jacobson radical. The ranks are closely related to the standard module.

Theorem 3.4 gives good information to consider the structure of standard modules. But it is not so easy to determine the structure of the standard module, in general. We restrict our attention only to the cases $(1) p \equiv 1(\bmod d)$ and $(2) d=3$.

\subsection{Case $p \equiv 1(\bmod d)$}

Let $K$ be an algebraically closed field of characteristic $p$. Suppose that $p \equiv 1(\bmod d)$. By Theorem 3.7, the adjacency algebra $K \operatorname{Cyc}\left(p^{a}, d\right)$ has basis $\left\{\boldsymbol{v}^{(i e)} \mid i=0,1, \ldots, d\right\}$, where $e=\left(p^{a}-1\right) / d$, and the multiplication is $\boldsymbol{v}^{(i e)} \boldsymbol{v}^{(j e)}=\boldsymbol{v}^{((i+j) e)}$ if $i+j \leq d$ and 0 otherwise. Also

$$
\left(\boldsymbol{v}^{(e)}\right)^{i}=\boldsymbol{v}^{(i e)}=\left(v_{1} v_{2} \cdots v_{a}\right)^{i(p-1) / d}
$$

for $i=0,1, \ldots, d$ and $\left(\boldsymbol{v}^{(e)}\right)^{d+1}=0$.

Proposition 4.1. We have $\operatorname{rank}_{K} \boldsymbol{v}^{(i e)}=(p-i(p-1) / d)^{a}$ for $i=0,1, \ldots, d$.

Proof. We can see that $\operatorname{rank}_{K} \boldsymbol{v}^{(i e)}=\operatorname{dim}_{K}(K N) \boldsymbol{v}^{(i e)}$ and $(K N) \boldsymbol{v}^{(i e)}$ has a basis

$$
\left\{v_{1}^{\ell_{1}} \cdots v_{a}^{\ell_{a}} \mid i(p-1) / d \leq \ell_{j} \leq p-1(j=1, \ldots, a)\right\} .
$$

So $\operatorname{rank}_{K} \boldsymbol{v}^{(i e)}=(p-i(p-1) / d)^{a}$.

We remark that $\operatorname{rank}_{K} \boldsymbol{v}^{(d e)}=1$.

We determine the structure of the standard module $K N$. Since $K S=(K N)^{H_{d}} \cong$ $K[x] /\left(x^{d+1}\right)$, indecomposable $K S$-modules are uniserial of length 1 to $d+1$ (corresponding to the Jordan normal form of $\left.\boldsymbol{v}^{(e)}\right)$. We denote them by $U_{j}(j=1, \ldots, d+1)$. Also we denote the multiplicity of $U_{j}$ in the standard module $K N$ by $m_{j}$. Namely

$$
K N \cong \bigoplus_{j=1}^{d+1} m_{j} U_{j}
$$

Since $\boldsymbol{v}^{(i e)}$ has rank $j-i$ on $U_{j}(j>i)$, we have

$$
\left(p-\frac{i(p-1)}{d}\right)^{a}=\operatorname{rank}_{K} \boldsymbol{v}^{(i e)}=\sum_{j=i+1}^{d+1}(j-i) m_{j} .
$$

The following theorem holds. 
Theorem 4.2. Let $K$ be an algebraically closed field of characteristic $p$. Suppose $p \equiv 1$ $(\bmod d)$. Then $K \operatorname{Cyc}\left(p^{a}, d\right) \cong K[x] /\left(x^{d+1}\right)$. We denote by $U_{j}$ the uniserial $K \operatorname{Cyc}\left(p^{a}, d\right)$ module of length $j$ for $j=1,2, \ldots, d+1$. Then $K X \cong \bigoplus_{j=1}^{d+1} m_{j} U_{j}$, where the multiplicities $m_{j}$ are determined by equations

$$
\left(p-\frac{i(p-1)}{d}\right)^{a}=\sum_{j=i+1}^{d+1}(j-i) m_{j} \quad(i=1, \ldots, d) .
$$

Remark that always $m_{d+1}=1$. If $a=1$, then we can compute all $m_{j}$.

Corollary 4.3. Suppose $a=1$ in Theorem 4.2. Then $m_{1}=\cdots=m_{d-1}=0, m_{d}=$ $(p-1) / d-1$, and $m_{d+1}=1$.

Proof. We know $m_{d+1}=1$. By $(p-(d-1)(p-1) / d)=m_{d}+2 m_{d+1}$, we have $m_{d}=$ $(p-1) / d-1$. Then $d m_{d}+(d+1) m_{d+1}=p=\operatorname{dim}_{K} K N$ and so $m_{1}=\cdots=m_{d-1}=0$.

We can determine ranks of adjacency matrices. The $p$-rank means the rank of a matrix over a field of characteristic $p$.

Corollary 4.4. Let $(X, S)=\operatorname{Cyc}\left(p^{a}, d\right)$ be a cyclotomic scheme with $p \equiv 1(\bmod d)$. For $s \in S \backslash\{1\}$, the $p$-rank of $\left(\sigma_{s}-n_{s} \sigma_{1}\right)^{i}$ is $(p-i(p-1) / d)^{a}$ for $i=0,1, \ldots, d$.

Proof. We know that $\left\{\sigma_{s}-n_{s} \sigma_{1} \mid s \in S \backslash\{1\}\right\}$ is a basis of the Jacobson radical of $K S$, where $K$ is a field of characteristic $p$. So $\sigma_{s}-n_{s} \sigma_{1} \in J(K S) \backslash J^{2}(K S)$ for some $s \in S \backslash\{1\}$. Then $\operatorname{rank}_{K}\left(\sigma_{s}-n_{s} \sigma_{1}\right)^{i}=\operatorname{rank}_{K}\left(\boldsymbol{v}^{(e)}\right)^{i}(i=0,1, \ldots, d)$. By Lemma 2.1, $\operatorname{rank}_{K}\left(\sigma_{s}-n_{s} \sigma_{1}\right)^{i}=\operatorname{rank}_{K}\left(\boldsymbol{v}^{(e)}\right)^{i}$ for every $s \in S \backslash\{1\}$.

If $d=2$ in Corollary 4.4, the result is just [3, Proposition in 4.1]. If $d=2$ and $a=1$, then the ranks are the same for all symmetric association schemes algebraically isomorphic to $\operatorname{Cyc}(p, 2)$ [9, Corollary 3.1].

Example 4.5 (Association schemes algebraically isomorphic to Cyc $\left.\left(5^{2}, 2\right)\right)$. There are 8 isomorphism classes of association schemes algebraically isomorphic to $\mathrm{Cyc}\left(5^{2}, 2\right)$ (order 25$, No. 4 to 11 in the list $[6])$. Let $(X, S)$ be one of them. Set $w=\sigma_{s}-12 \sigma_{1}$ for some $s \in S \backslash\{1\}$. Then $\operatorname{rank}(w)=12$ for No. 4, 5, 6, 7, 8, $\operatorname{rank}(w)=11$ for No. 9, 10, and $\operatorname{rank}(w)=9$ for No. 11. By Corollary 4.4, the cyclotomic scheme Cyc $\left(5^{2}, 2\right)$ is No. 11 . It is characterized by $\operatorname{rank}(w)$ and has the smallest value, in this case.

\subsection{Case $d=3$}

We consider $(X, S)=\operatorname{Cyc}\left(p^{a}, 3\right)$. If $p \equiv 1(\bmod 3)$, then we can apply results in Subsection 4.1. We assume that $p \equiv 2(\bmod 3)$. Remark that $a$ is even. Then $K \mathrm{Cyc}\left(p^{a}, 3\right) \cong K[x, y] /\left(x^{2}, y^{2}\right)$ by Theorem 3.10. There are infinitely many indecomposable modules and they are classified (see $[2,4.3]$, for example). We put

$$
\alpha=\frac{2 p-1}{3}, \quad \beta=\frac{p-2}{3}
$$


and

$$
x=\boldsymbol{v}^{(e)}=\prod_{i=1}^{a / 2}\left(v_{2 i-1}^{\alpha} v_{2 i}^{\beta}\right), \quad y=\boldsymbol{v}^{(2 e)}=\prod_{i=1}^{a / 2}\left(v_{2 i-1}^{\beta} v_{2 i}^{\alpha}\right) .
$$

To describe results, we use diagrams like the following.

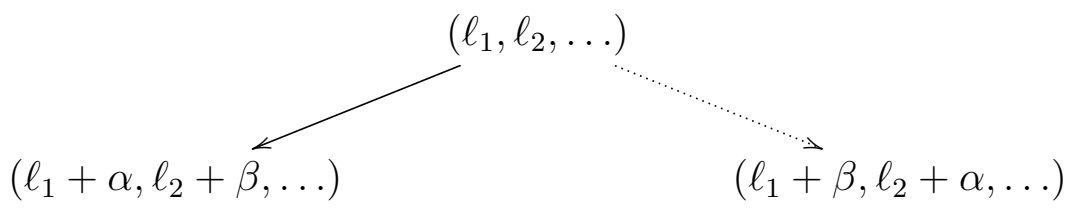

In the diagram, $\left(\ell_{1}, \ell_{2}, \ldots\right)$ means $v_{1}^{\ell_{1}} v_{2}^{\ell_{2}} \ldots$, the arrow means multiplying $x$, and the dotted arrow means multiplying $y$. The diagram defines a representation of $K \operatorname{Cyc}\left(p^{a}, d\right)$. Take a basis $v_{1}^{\ell_{1}} v_{2}^{\ell_{2}} \cdots, v_{1}^{\ell_{1}+\alpha} v_{2}^{\ell_{2}+\beta} \cdots, v_{1}^{\ell_{1}+\beta} v_{2}^{\ell_{2}+\alpha} \cdots$. Then the representation is

$$
x \mapsto\left(\begin{array}{ccc}
0 & 1 & 0 \\
0 & 0 & 0 \\
0 & 0 & 0
\end{array}\right), y \mapsto\left(\begin{array}{ccc}
0 & 0 & 1 \\
0 & 0 & 0 \\
0 & 0 & 0
\end{array}\right) .
$$

We define six modules (representations) :

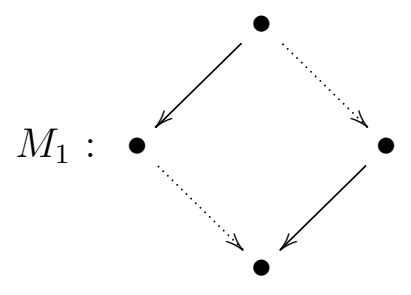

$$
\begin{gathered}
M_{1}: \bullet \mapsto\left(\begin{array}{llll}
0 & 1 & 0 & 0 \\
0 & 0 & 0 & 0 \\
0 & 0 & 0 & 1 \\
0 & 0 & 0 & 0
\end{array}\right), y \mapsto\left(\begin{array}{lll}
0 & 1 & 0 \\
0 & 0 & 0 \\
0 & 0 & 0
\end{array}\right), y \mapsto\left(\begin{array}{llll}
0 & 0 & 1 & 0 \\
0 & 0 & 0 & 1 \\
0 & 0 & 0 & 0 \\
0 & 0 & 0 & 0
\end{array}\right) \\
M_{2}:
\end{gathered}
$$

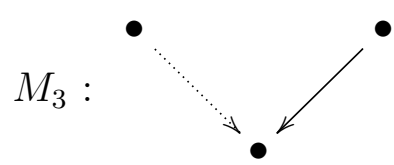

$$
x \mapsto\left(\begin{array}{ccc}
0 & 0 & 1 \\
0 & 0 & 0 \\
0 & 0 & 0
\end{array}\right), y \mapsto\left(\begin{array}{ccc}
0 & 0 & 0 \\
0 & 0 & 1 \\
0 & 0 & 0
\end{array}\right)
$$

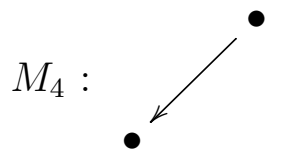

$$
x \mapsto\left(\begin{array}{cc}
0 & 1 \\
0 & 0
\end{array}\right), y \mapsto\left(\begin{array}{ll}
0 & 0 \\
0 & 0
\end{array}\right)
$$

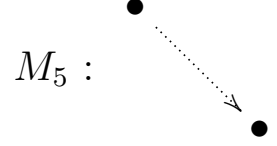

$$
x \mapsto\left(\begin{array}{ll}
0 & 0 \\
0 & 0
\end{array}\right), y \mapsto\left(\begin{array}{ll}
0 & 1 \\
0 & 0
\end{array}\right)
$$

$M_{6}$ :

$$
x \mapsto(0), y \mapsto(0)
$$


All of the modules (representations) above are indecomposable by [2, 4.3]. The vertices move over the range $0 \leq \ell_{i} \leq p-1(1 \leq i \leq a)$. If we take a connected diagram as large as possible, then the vector space spanned by the elements corresponding to the vertices is an $K S$-submodule of the standard module $K X$. So if we decompose all vertices into connected diagrams, we can get the indecomposable direct sum decomposition of $K X$. It is easy to see that only the six diagrams above are possible. For example, the diagram

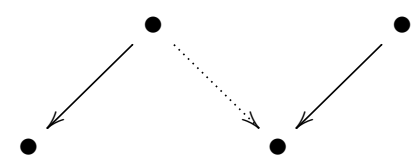

is impossible, because if we put the lower left vertex $\left(\ell_{1}, \ell_{2}, \ldots\right)$, then the upper right vertex is $\left(\ell_{1}-p, \ell_{2}+1, \ldots\right)$, but $0 \leq \ell_{1}, \ell_{1}-p \leq p-1$ is impossible.

Now we can determine the structure of the standard module.

Theorem 4.6. Let $K$ be an algebraically closed field of characteristic $p$. Suppose $p \equiv 2$ $(\bmod 3)$. Then $K \mathrm{Cyc}\left(p^{a}, d\right) \cong K[x, y] /\left(x^{2}, y^{2}\right)$. The standard module is $\bigoplus_{i=1}^{6} m_{i} M_{i}$, where

$$
\begin{aligned}
& m_{1}=1, \quad m_{2}=m_{3}=\frac{(p+1)^{a}}{3^{a}}-1, \\
& m_{4}=m_{5}=\frac{\left(2^{a / 2}-2\right)(p+1)^{a}}{3^{a}}, \quad m_{6}=p^{a}+2-\frac{\left(2^{a / 2+2}-2\right)(p+1)^{a}}{3^{a}} .
\end{aligned}
$$

Proof. Obviously, there is only one summand $M_{1}$ with the top vertex $(0, \ldots, 0)$. The vertex $\left(\ell_{1}, \ldots, \ell_{a}\right)$ is a top vertex of $M_{2}$ if and only if $0 \leq \ell_{i} \leq \alpha$ for all $i=1,2, \ldots, a$ except the case $M_{1}$. So $m_{2}=(\alpha+1)^{a}-1=(p+1)^{a} / 3^{a}-1$. By symmetry, $m_{3}=m_{2}$. The vertex $\left(\ell_{1}, \ldots, \ell_{a}\right)$ is a top vertex of $M_{4}$ if and only if $0 \leq \ell_{2 i-1} \leq \alpha, 0 \leq \ell_{2 i} \leq \beta$ for all $i=1,2, \ldots, a / 2$ except the cases $M_{1}, M_{2}, M_{3}$. So

$$
m_{4}=(\alpha+1)^{a / 2}(\beta+1)^{a / 2}-2-2\left(\left(\alpha^{a}+1\right)-1\right)=\left(\frac{p+1}{3}\right)^{a}\left(2^{a / 2}-2\right) .
$$

By symmetry, we have $m_{5}=m_{4}$. Counting the number of all vertices, we have $m_{6}$.

We determine the $p$-rank of $\sigma_{s}-n_{s} \sigma_{1}$ for $s \in S \backslash\{1\}$.

Corollary 4.7. Let $(X, S)$ be the cyclotomic scheme Cyc $\left(p^{a}, 3\right)$ with $p \equiv 2(\bmod 3)$. Then, for $s \in S \backslash\{1\}$, the $p$-rank of $\sigma_{s}-n_{s} \sigma_{1}$ is $2^{a / 2}(p+1)^{a} / 3^{a}$.

Proof. Note that $J^{2}(K S)=K \sum_{t \in S} \sigma_{t}=K x y$ and $\sigma_{s}-n_{s} \sigma_{1} \in J(K S) \backslash J^{2}(K S)$. So we can write $\sigma_{s}-n_{s} \sigma_{1}=c_{x} x+c_{y} y+c_{x y} x y$ with $c_{x}, c_{y}, c_{x y} \in K,\left(c_{x}, c_{y}\right) \neq(0,0)$. For the representation of $M_{1}$, we have

$$
\sigma_{s}-n_{s} \sigma_{1} \mapsto\left(\begin{array}{cccc}
0 & c_{x} & c_{y} & c_{x y} \\
0 & 0 & 0 & c_{y} \\
0 & 0 & 0 & c_{x} \\
0 & 0 & 0 & 0
\end{array}\right)
$$


and the rank is 2. Similarly we have the rank 1 for $M_{2}, M_{3}$, and 0 for $M_{6}$. Since $m_{4}=m_{5}$, consider the sum $M_{4} \oplus M_{5}$,

$$
\sigma_{s}-n_{s} \sigma_{1} \mapsto\left(\begin{array}{cccc}
0 & c_{x} & 0 & 0 \\
0 & 0 & 0 & 0 \\
0 & 0 & 0 & c_{y} \\
0 & 0 & 0 & 0
\end{array}\right)
$$

The rank for this representation is 1 if $c_{x}=0$ or $c_{y}=0$ and 2 otherwise. Since the ranks of $\sigma_{t}-n_{t} \sigma_{1}$ are constant for $t \in S \backslash\{1\}$ by Lemma 2.1 and $|S|=4$, we can see that the rank is 2. By Theorem 4.6, the rank of $\sigma_{s}-n_{s} \sigma_{1}$ for the standard representation is

$$
2+2\left(\frac{(p+1)^{a}}{3^{a}}-1\right)+2\left(2^{a / 2}-2\right) \frac{(p+1)^{a}}{3^{a}}=\frac{2^{a / 2}(p+1)^{a}}{3^{a}}
$$

and we have the result.

Example 4.8 (Association schemes algebraically isomorphic to $\mathrm{Cyc}\left(2^{4}, 3\right)$ ). In the list in [6], Order 16 No. 20 is the cyclotomic scheme $\mathrm{Cyc}\left(2^{4}, 3\right)$ and No. 21 is a non-schurian scheme algebraically isomorphic to $\operatorname{Cyc}\left(2^{4}, 3\right)$. For both cases, $\operatorname{rank}_{K}\left(\sigma_{s}-n_{s} \sigma_{s}\right)=$ $\operatorname{dim}_{K} K X\left(\sigma_{s}-n_{s} \sigma_{s}\right)=6$ for any $s \in S \backslash\{1\}$. But for $s, t \in S \backslash\{1\}, s \neq t$, $\operatorname{dim}_{K}(K X) J(K S)=\operatorname{dim}_{K}\left(K X\left(\sigma_{s}-n_{s} \sigma_{s}\right)+K X\left(\sigma_{t}-n_{s} \sigma_{t}\right)\right)=7$ for No. 20 and 8 for No. 21. The difference comes from the structure of the standard modules. For the cyclotomic scheme No. 20, we can compute the dimension of $K X\left(\sigma_{s}-n_{s} \sigma_{s}\right)+K X\left(\sigma_{t}-n_{s} \sigma_{t}\right)$ to be 7 by Theorem 4.6. The standard module is $M_{1} \oplus 2 M_{4} \oplus 2 M_{5} \oplus 4 M_{6}$, and $\operatorname{dim}_{K}\left(M_{1}\left(\sigma_{s}-n_{s} \sigma_{s}\right)+M_{1}\left(\sigma_{t}-n_{s} \sigma_{t}\right)\right)=3, \operatorname{dim}_{K}\left(M_{4}\left(\sigma_{s}-n_{s} \sigma_{s}\right)+M_{4}\left(\sigma_{t}-n_{s} \sigma_{t}\right)\right)=1$, $\operatorname{dim}_{K}\left(M_{5}\left(\sigma_{s}-n_{s} \sigma_{s}\right)+M_{5}\left(\sigma_{t}-n_{s} \sigma_{t}\right)\right)=1$, and $\operatorname{dim}_{K}\left(M_{6}\left(\sigma_{s}-n_{s} \sigma_{s}\right)+M_{6}\left(\sigma_{t}-n_{s} \sigma_{t}\right)\right)=0$. So $K X\left(\sigma_{s}-n_{s} \sigma_{s}\right)+K X\left(\sigma_{t}-n_{s} \sigma_{t}\right)=7$.

Example 4.9 (Association schemes algebraically isomorphic to $\mathrm{Cyc}\left(5^{2}, 3\right)$ ). In the list in [6], Order 25 No. 18 is the cyclotomic scheme $\operatorname{Cyc}\left(5^{2}, 3\right)$ and No. 17 is a schurian scheme algebraically isomorphic to $\operatorname{Cyc}\left(5^{2}, 3\right)$. For both cases, $\operatorname{rank}_{K}\left(\sigma_{s}-n_{s} \sigma_{s}\right)=$ $\operatorname{dim}_{K} K X\left(\sigma_{s}-n_{s} \sigma_{s}\right)=8$ for any $s \in S \backslash\{1\}$. But $\operatorname{dim}_{K}\left(\bigcap_{s \in S \backslash\{1\}} K X\left(\sigma_{s}-n_{s} \sigma_{1}\right)\right)=3$ for No. 17 and 4 for No. 18. The difference comes from the structure of the standard modules. For the cyclotomic scheme No. 18, we can compute the dimension to be 4 by Theorem 4.6. The standard module is $M_{1} \oplus 3 M_{2} \oplus 3 M_{3} \oplus 3 M_{6}$, and $\operatorname{dim}_{K}\left(\bigcap_{s \in S \backslash\{1\}} M_{1}\left(\sigma_{s}-\right.\right.$ $\left.\left.n_{s} \sigma_{1}\right)\right)=1, \operatorname{dim}_{K}\left(\bigcap_{s \in S \backslash\{1\}} M_{2}\left(\sigma_{s}-n_{s} \sigma_{1}\right)\right)=0, \operatorname{dim}_{K}\left(\bigcap_{s \in S \backslash\{1\}} M_{3}\left(\sigma_{s}-n_{s} \sigma_{1}\right)\right)=1$, and $\operatorname{dim}_{K}\left(\bigcap_{s \in S \backslash\{1\}} M_{6}\left(\sigma_{s}-n_{s} \sigma_{1}\right)\right)=0$. So $\operatorname{dim}_{K}\left(\bigcap_{s \in S \backslash\{1\}} K X\left(\sigma_{s}-n_{s} \sigma_{1}\right)\right)=4$.

\section{Acknowledgments}

The author would like to thank the referee and the editor for their comments.

\section{References}

[1] E. Bannai and T. Ito, Algebraic combinatorics. I, The Benjamin/Cummings Publishing Co. Inc., Menlo Park, CA, 1984. 
[2] D. J. Benson, Representations and cohomology. I, Cambridge Studies in Advanced Mathematics, vol. 30, Cambridge University Press, Cambridge, 1991.

[3] A. E. Brouwer and C. A. van Eijl, On the p-rank of the adjacency matrices of strongly regular graphs, J. Algebraic Combin. 1 (1992), no. 4, 329-346.

[4] R. W. Goldbach and H. L. Claasen, Cyclotomic schemes over finite rings, Indag. Math. (N.S.) 3 (1992), no. 3, 301-312.

[5] A. Hanaki, Locality of a modular adjacency algebra of an association scheme of prime power order, Arch. Math. (Basel) 79 (2002), no. 3, 167-170.

[6] A. Hanaki and I. Miyamoto, Classification of association schemes with small vertices, published on web (http://math.shinshu-u.ac.jp/ ${ }^{\sim}$ hanaki/as/).

[7] A. Hanaki and M. Yoshikawa, On modular standard modules of association schemes, J. Algebraic Combin. 21 (2005), no. 3, 269-279.

[8] T. Kasami, S. Lin, and W. W. Peterson, Some results on cyclic codes which are invariant under the affine group and their applications, Information and Control 11 (1967), 475-496.

[9] R. Peeters, Uniqueness of strongly regular graphs having minimal p-rank, Linear Algebra Appl. 226/228 (1995), 9-31. MR 1344551

[10] On the p-ranks of the adjacency matrices of distance-regular graphs, J. Algebraic Combin. 15 (2002), no. 2, 127-149.

[11] O. Shimabukuro and M. Yoshikawa, Modular adjacency algebras of Grassmann graphs, Linear Algebra Appl. 466 (2015), 208-217.

[12] M. Yoshikawa, Modular adjacency algebras of Hamming schemes, J. Algebraic Combin. 20 (2004), no. 3, 331-340.

[13] _ The modular adjacency algebras of non-symmetric imprimitive association schemes of class 3, J. Fac. Sci. Shinshu Univ. 40 (2005), 27-36 (2006).

[14] P.-H. Zieschang, An algebraic approach to association schemes, Lecture Notes in Mathematics, vol. 1628, Springer-Verlag, Berlin, 1996. 